\title{
Laboratory nutritional parameters can predict one-year mortality in elderly patients with intertrochanteric fracture
}

\author{
Jun Lu' ${ }^{1 *}$, Yuan-yuan Chen ${ }^{1}$, Lin Zhang ${ }^{1}$, Xin-ying Wang ${ }^{2}$, Yong-gang Lii ${ }^{1}$ and Chen Wang ${ }^{1}$ \\ ${ }^{1}$ Department of Orthopaedic Surgery, Zhongda Hospital, Southeast University, 87 Dingjiaqiao Road 210009, Nanjing, Jiangsu province, China \\ ${ }^{2}$ Department of General Surgery, Jinling Hospital, Medical School, Nanjing University, 305 Zhongshan East Road 210002, Nanjing, Jiangsu province, China
}

Received: March 20, 2014; Accepted: April 26, 2014; Published: May 07, 2014

*Corresponding author: Jun Lu, Department of Orthopaedic Surgery, Zhongda Hospital, Southeast University, 87 Dingjiaqiao Road 210009, Nanjing, Jiangsu province, China. Tel: +86 258326 2340; Fax: +86 258326 2342; Email: lujun@medmail.com.cn

\begin{abstract}
Objectives: The purpose of this study was to investigate the contributing value of nutrition related blood parameters to one-year mortality following intertrochanteric fracture surgery in a Chinese population over the age of 65 .

Methods: The nutritional status was evaluated by using admission serum albumin level and total lymphocyte count (TLC). One hundred and seventy-four intertrochanteric fracture patients were entered to this study for nutritional status assessment. Gender differences in these parameters were evaluated by univariate analysis. The predictive value of the variables to one-year mortality was identified by multiple logistic regression analysis.

Results: The mean albumin level was $31.0 \mathrm{~g} / \mathrm{L}$ with $73 \%$ of patients less than $35 \mathrm{~g} / \mathrm{L}$ and the mean TLC was $1.19 \times 10^{9} \mathrm{cells} / \mathrm{L}$ with $81 \%$ less than $1.50 \times 10^{9} \mathrm{cells} / \mathrm{L}$. There were no significant differences in albumin $(p=0.674)$ or TLC $(p=0.804)$ level between male and female patients. Survival information was obtained in 149 patients who received surgical treatment. The general one-year mortality was $31 \%$ with $35 \%$ in men and $29 \%$ in women, respectively. The surviving patients were younger and had higher albumin, TLC and calcium levels than those who died. However, multivariate analysis identified only serum albumin and TLC as the independent and significant risk factors associated with one-year mortality; the optimal cut-off point is $29.5 \mathrm{~g} / \mathrm{L}$ and $0.93 \times 10^{9}$ cells/L, respectively, based on the receiver operating characteristic (ROC) curve.(Koren-Hakim, Weiss et al. 2012)
\end{abstract}

Conclusions: Malnutrition is a common phenomenon in an aged Chinese population suffering from intertrochanteric fractures. A lower serum albumin level and total lymphocyte count at admission are significant risk factors to predict the one-year mortality.

Keywords: Nutritional status; Intertrochanteric fractures; Mortality; Elderly people

\section{Abbreviations}

TLC: Total Lymphocyte Count; ROC curve: Receiver Operating Characteristic Curve; GN: Gamma Nail; PFN: Proximal Femoral Nail; DHS: Dynamic Hip Screw

\section{Introduction}

Intertrochanteric fracture, a subset of hip fracture, is one of the most common injuries in elderly people and, together with femoral neck fracture, presents perhaps the most important public health problem facing orthopaedic surgeons today. Aged people with hip fracture are at considerable risk for premature death [1-5] with increased mortality during the first year after surgery from $8.4 \%$ to $36 \%$ [2]. Although numerous studies have focused on the risk factors for mortality following hip fracture surgery, there is no conclusive evidence yet what preoperative factors predict postoperative mortality [6].

Elderly patients with hip fracture are more likely malnourished on admission when compared to the age-matched general population [7-9]. The poor nutritional status is known to be both a common causative factor of hip fracture and a predictor of excess mortality following surgical intervention [10, 11]. Many methods including anthropometry, the mini-nutritional assessment, nutrition related blood parameters and dietary analysis are used for nutritional status assessment in clinic [12, 13]. Serum albumin and total lymphocyte count (TLC) are two of the most important blood markers for nutritional status [14] and have been recognized as prognostic factors of hip fractures [15-18]. Several studies have demonstrated serum albumin [14, $16,17,19-21]$ or TLC $[16,17,22]$ on admission as an independent predictor of postoperative mortality in aged people with hip fracture. However, most of the studies have not analyzed the different fracture types separately, although the fracture type per se may be influenced by nutrition status [23] and may be predictive of mortality [24].

The purpose of this study is to assess the nutritional status of intertrochanteric fracture patients from a Chinese population aged over 65 years by preoperative serum albumin and total lymphocyte count level. The relationship between these blood markers and the patients' one-year mortality is also analyzed.

\section{Materials and Methods}

\section{Study design and data collection}

All patients were admitted to a university affiliated hospital with an acute intertrochanteric fracture over a 24-month period (January 2009-December 2010) and studied retrospectively. 
This study was approved by the Ethics Committee of Southeast University affiliated Zhongda Hospital. The basic information of the patients (such as name and identification number) was searched by the diagnosis of intertrochanteric fracture from a discharge registration system. Then the original medical records which contain all laboratory test results of the inpatients were retrieved from the record room of the hospital. Patients younger than 65 years of age, with multiple fractures or malignant pathologic fracture, and those not managed operatively were excluded from the study.

The demographic data (age and gender) and five preoperative blood parameters (serum albumin, TLC, hemoglobin, serum potassium and calcium) were entered into a confidential database (MS Excel ${ }^{\mathrm{TM}}$, Microsoft Corp, Seattle, WA). Admission serum albumin and TLC results were used as the sole markers of nutritional status. Values of serum albumin $(<35 \mathrm{~g} / \mathrm{L})$ and TLC $\left(<1.5 \times 10^{9}\right.$ cells $/$ L $)$ below the lower laboratory reference range were taken to represent malnutrition $[14,16]$. The proportion of patients with either or both parameters below the normal level was calculated and gender differences in age, blood parameters and mortality were analyzed.

Fracture type and surgical intervention were confirmed according to the operation records. As intramedullary fixation is the first choice of surgical treatment for intertrochanteric fractures in our hospital, a majority of the fractures were stabilized with the gamma nail (GN) or the proximal femoral nail (PFN). The operating procedures were similar, the main difference being the type of implant [25]. All surgeries were performed according to the standard protocols of either GN or PFN by experienced surgeons. Fractures treated with other fixation methods, for example Dynamic hip screw (DHS), were excluded from the study.

As a national database of population death registration system has not been established in China, a telephone follow-up survey was performed one year after the surgery for each case. If the patient was unavailable, a family member or direct caregiver was contacted instead. If the telephone number in the medical record could not get through, a home visit was made according to the family address in the record. If the patient's survival information still could not be obtained, the patient was excluded from the mortality analysis.

\section{Statistical analysis}

Continuous variables were described as average \pm standard deviation. Categorical variables were presented in terms of their frequency. The gender difference in one-year mortality was tested using Pearson Chi squared method. The independent sample T test was used to compare the mean level of blood parameters between male and female patients. A univariate analysis of variance was performed on data form patients who survived one year postoperatively and on data from those who did. The relative contribution of the variables to mortality was identified using a multiple logistic regression analysis with a forward stepwise variable selection. The results of multivariate analyses were expressed in terms of hazard ratio derived from the estimated regression coefficients, with $95 \%$ confidence intervals. Statistical analysis was performed using the Statistical Package for the Social Sciences 17.0 version for Windows program (SPSS Inc, Chicago, IL, USA). P-values $<0.05$ were considered significant. The optimal cut-off points of the significant factors were determined using receiver operating characteristic (ROC) curves [26]. This involved plotting the true-positive rate (sensitivity) against the false-positive rate (1-specificity) for possible cut-off scores. Each point on the ROC plot represented a sensitivity/specificity pair corresponding to a particular decision threshold. The highest sensitivity/specificity combination point on the ROC curve represents the optimal cut-off point of the predictors.

\section{Results}

One hundred and seventy four patients with intertrochanteric fracture over the age of 65 years were admitted during the study period. Nutritional and demographic data were available for all 174 patients, of whom 116 (67\%) were women and 58 $(33 \%)$ were men. The gender differences in mean age and blood parameters at admission are shown in table 1 . The only significant difference index is hemoglobin where the level of hemoglobin in male patients is higher than that in female patients. The mean albumin for all patients was $31.0 \pm 5.3 \mathrm{~g} / \mathrm{L}$ (range: 18 to 43 ; median 31) with an albumin level less than $35.0 \mathrm{~g} / \mathrm{L}$ occurred in $127(73 \%)$ patients. The mean TLC was $1.19 \pm 0.51 \times 10^{9}$ cells/L (range: 0.29 to $3.47 \times 10^{9}$ cells $/$ L; median $1.1 \times 10^{9}$ cells $/$ L) with 141 $(81 \%)$ patients lower than $1.50 \times 10^{9}$ cells/L. Fifty two percent of the patients had both albumin and TLC levels lower than normal.

Seven patients receiving non operative treatment and two patients treated with DHS stabilization were excluded from the mortality study. Sixteen patients were lost to follow up one year after surgery leaving survival data for 149 patients $(86 \%)$. The mean age of the patients was $80.9 \pm 7.5$ (range: 65 to 97; median 82). Females ( $n=98 ; 34 \%)$ outnumbered males $(n=51 ; 66 \%)$. Forty six patients died within one year postoperatively and the overall mortality rate was $31 \%$. Two patients died of pulmonary infection during their hospital stay and both of them had low albumin and TLC levels at admission. One hundred eleven (74\%) patients were malnourished based on albumin levels less than $35.0 \mathrm{~g} / \mathrm{L}$ of whom $45(41 \%)$ died. Only one patient (3\%) died with admission albumin $>35.0 \mathrm{~g} / \mathrm{L}(\mathrm{p}<0.001)$. There was no difference in one-year mortality between the patients $(23 \%$,

Table 1: Univariate analysis comparing the age and blood parameters between elderly male and female patients with intertrochanteric fracture.

\begin{tabular}{|c|c|c|c|}
\hline Variable & male & female & p-value \\
\hline Age & $79.39 \pm 7.34$ & $81.76 \pm 7.46$ & 0.067 \\
\hline Serum albumin (g/L) & $31.14 \pm 5.70$ & $30.76 \pm 5.00$ & 0.674 \\
\hline $\begin{array}{c}\text { Totle lymphocyte count } \\
\left(\times 10^{9} / \mathrm{L}\right)\end{array}$ & $1.16 \pm 0.48$ & $1.18 \pm 0.55$ & 0.804 \\
\hline Haemoglobin (g/L) & $107.20 \pm 20.13$ & $100.18 \pm 13.17$ & 0.027 \\
\hline $\begin{array}{c}\text { Serum potassium } \\
\text { (mmol/L) }\end{array}$ & $3.93 \pm 0.40$ & $3.84 \pm 0.45$ & 0.255 \\
\hline Serum calcium (mmol/L) & $2.13 \pm 0.12$ & $2.12 \pm 0.14$ & 0.975 \\
\hline
\end{tabular}

Variables were reported as median \pm standard deviation 
$6 / 26)$ with TLC $\geq 1.50 \times 10^{9}$ cells/L and those $(31 \%, 40 / 123)$ with $\mathrm{TLC}<1.50 \times 10^{9}$ cells $/ \mathrm{L}(\mathrm{p}=0.344)$. Demographic characteristics and blood parameters of the survivors and non-survivors are summarized in table 2. Variables comparison between the two groups (survival at one year post surgery or not) found that age $(p=0.018)$, serum albumin level $(p<0.001)$, TLC level $(p=0.006)$ and serum calcium level $(\mathrm{p}=0.010)$ had significant differences. However, multiple logistic regression analysis with stepwise variable selection showed that only serum albumin level ( $\mathrm{p}<0.001$, hazard ratio 1.223) and TLC level $(\mathrm{p}=0.008$, hazard ratio 3.899) at hospital admission had significant association with one-year mortality (table 3).

Based on the ROC curves, the optimal cut-off points for serum albumin level and TLC level were $29.5 \mathrm{~g} / \mathrm{L}$ (sensitivity $76.7 \%$; specificity $65.2 \%$ ) and $0.925 \times 10^{9}$ cells/L (sensitivity $77.7 \%$; specificity $58.7 \%$ ) respectively (Figure 1 ). If the patients were classified based on their albumin level at $30.0 \mathrm{~g} / \mathrm{L}$, the one-year mortality in patients with albumin equal or higher than $30.0 \mathrm{~g} / \mathrm{L}$ $(16.8 \%, 16 / 95)$ was significantly lower than those below $30.0 \mathrm{~g} / \mathrm{L}$ $(55.6 \%, 30 / 54, \mathrm{p}<0.001)$. When the patients were classified based on their TLC level at $1 \times 10^{9}$ cells $/ \mathrm{L}$, the mortality in patients with TLC equal or higher than $1.00 \times 10^{9}$ cells/L $(18.2 \%, 16 / 88)$ was significantly lower than those below $1.00 \times 10^{9} \mathrm{cells} / \mathrm{L}$ $(49.2 \%, 30 / 61, \mathrm{p}<0.001)$.

\section{Discussion}

The incidence of hip fracture is increasing, paralleling the increased longevity of the global population [27]. Almost 9 of 10 hip fractures occur in patients older than 65 years and approximately half will be intertrochanteric fractures. Many studies have investigated the mortality of hip fractures in elderly patients and most include all types of hip fractures grouped together. It is generally known that intertrochanteric fracture patients are slightly older and sicker than femoral neck fracture patients [28]. Survival studies focusing on fracture type have demonstrated that intertrochanteric fracture patients have a higher postoperative mortality rate than those with femoral neck fracture $[24,28,29]$. Since so many differences exist in patient characteristics, surgical management and outcomes between femoral neck fracture and intertrochanteric fracture, it is reasonable to single out the specific fracture group for risk predictor evaluation. Here, we focus on the relationship between the nutritional status and long term mortality following surgery in an aged Chinese patients group admitted with a femur intertrochanteric fracture. We found a high rate of malnutrition among these patients based on the serum albumin and TLC levels at admission, and we demonstrated that both of these parameters can significantly predict one-year mortality in this patients group.

The increased prevalence of poor nutritional status among older adults with hip fractures compared to the general population has been well documented [7-11,13,14,16,18-21]. Malnutrition combined with the catabolic response to trauma or surgery, leads to muscle wasting and has been associated with post-operative complications [18], increased length of stay $[15,17]$ and mortality [14,19-21]. However most of these studies were based on patients from developed western countries. The nutritional status of hip fractured patients from a developing country such as China with a different healthcare delivery system is still unclear. In the present study, we employed admission serum albumin and TLC level as markers of nutrition assessment. Both of them are routine preoperative laboratory tests in our hospital and numerous studies have used a combination of

Table 2: Univariate analysis comparing survivors and non-survivors 1 year after surgery in elderly intertrochanteric fracture patients.

\begin{tabular}{|l|c|c|}
\hline \multicolumn{1}{|c|}{ Variable } & die & p-value \\
\hline Gender, Male (\%) & $18(35.29 \%)$ & $33(64.71 \%)$ \\
\hline Age & $83.11 \pm 7.59$ & $79.98 \pm 7.25$ \\
\hline Serum albumin (g/L) & $27.72 \pm 4.15$ & $32.30 \pm 5.06$ \\
\hline Totle lymphocyte count $\left(\times 10^{9} / \mathrm{L}\right)$ & $1.00 \pm 0.45$ & 0.018 \\
\hline Haemoglobin (g/L) & $99.70 \pm 14.93$ & $1.26 \pm 0.54$ \\
\hline Serum potassium (mmol/L) & $3.95 \pm 0.50$ & $103.87 \pm 16.61$ \\
\hline Serum calcium (mmol/L) & $2.09 \pm 0.14$ & $3.84 \pm 0.40$ \\
\hline
\end{tabular}

Table 3: Multiple logistic regression analysis for on one-year mortality after surgery in elderly intertrochanteric fracture patients.

\begin{tabular}{|c|c|c|c|c|c|}
\hline Variable & Regression coefficient & Standard error & Test statistic & P value & $\begin{array}{c}\text { Hazard ratio } \\
(95 \% \mathrm{CI})\end{array}$ \\
\hline Gender (Male) & .554 & .442 & 1.570 & .210 & $1.740(0.732-4.135)$ \\
\hline Serum albumin (g/L) & .202 & .050 & 15.951 & .000 & $1.223(1.108-1.351)$ \\
\hline $\operatorname{TLC}\left(\times 10^{9} / \mathrm{L}\right)$ & 1.361 & .509 & 7.144 & .008 & $3.899(1.437-10.577)$ \\
\hline Serum potassium $(\mathrm{mmol} / \mathrm{L})$ & -.879 & .497 & 3.120 & .077 & $0.415(0.157-1.101)$ \\
\hline Serum calcium (mmol/L) & -.532 & 1.819 & .085 & .770 & $0.588(0.017-20.753)$ \\
\hline
\end{tabular}

$\mathrm{CI}=$ confidence interval, TLC=total lymphocyte count 

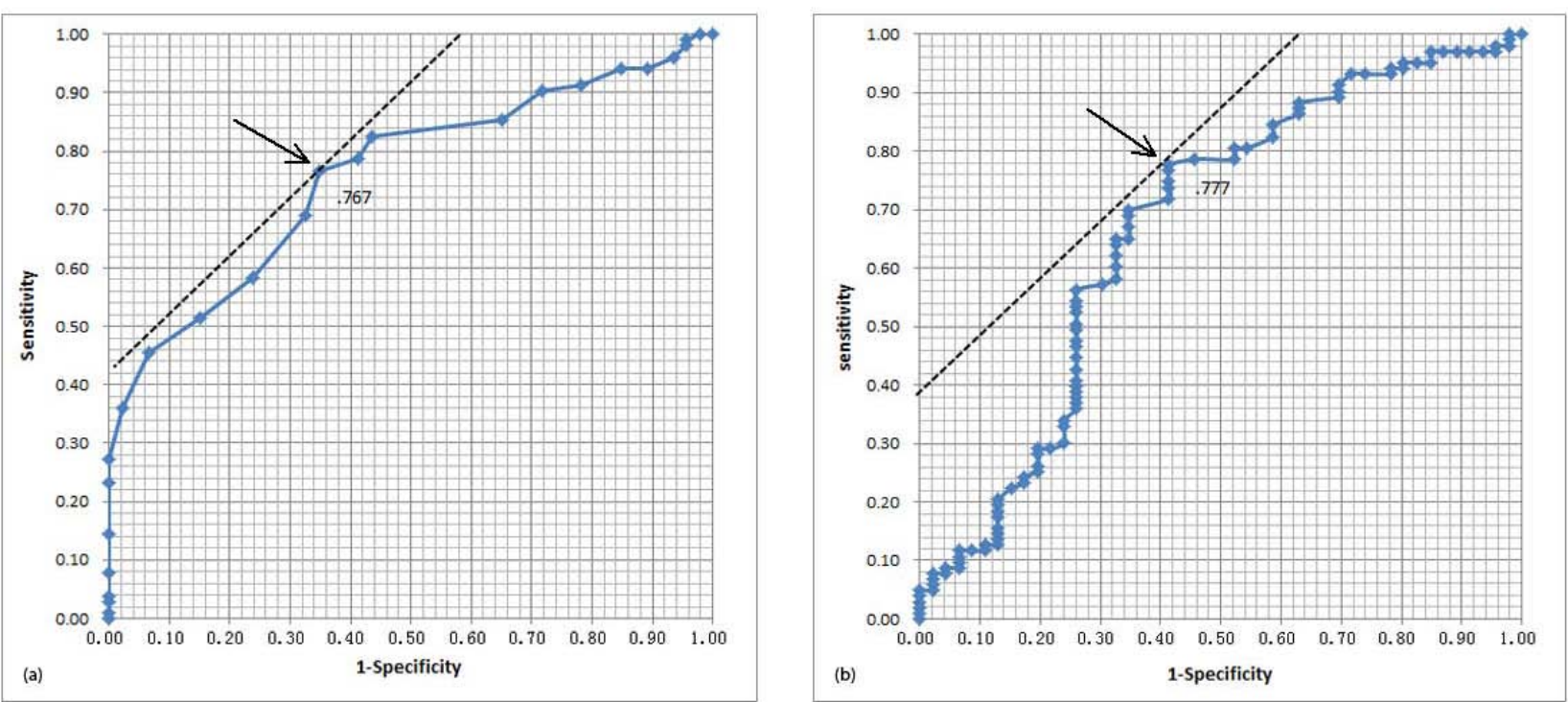

Figure 1: Receiver operating characteristic curves for (a) serum albumin level and (b) total lymphocyte count. Black arrows indicate the optimal cutoff points of $29.5 \mathrm{~g} / \mathrm{L}$ for serum albumin level and $0.925 \times 109$ cells $/ \mathrm{L}$ for total lymphocyte count corresponding to the highest sensitivity/specificity combination. Dotted line indicates reference line (0 diagnostic efficiency).

albumin and TLC levels to identify whether the patients are under a state of protein energy malnutrition $[14,16,19]$. According to the widely accepted reference value, $35.0 \mathrm{~g} / \mathrm{L}$ for albumin and $1.50 \times 10^{9}$ cells/L for TLC, we observed $73 \%$ of the patients with a low albumin level, $81 \%$ with a low TLC level and 52\% with both under the reference values. The incidence of malnutrition is much higher than that reported by Koval et al. They evaluated nutritional status of 490 hip fracture patients aged 65 years and older in the United States, and reported 18\% patients with low albumin, 57\% with low TLC, and only $9 \%$ having both parameters below the normal value [16]. More recently, another study from Ireland using serum albumin and TLC as the sole markers in hip fracture patients aged over 60 years, and reported $66 \%$ patients with low albumin level, $74 \%$ with low TLC level, and $51 \%$ with both low albumin and TLC levels [26]. The proportion of intertrochanteric fracture in both studies was about $50 \%$, but neither had analyzed the difference of nutritional status by fracture type. Although the data from Ireland are comparable to the present study, we admit that the regional difference of nutritional status in aged people is unavoidable because of the inconsistency in economy, culture, hygiene and diet. We did not find any gender differences in age or nutritional status and it is not surprising that male patients have a higher mean heamoglobin level than women. Because previous studies have demonstrated a relationship between low heamoglobin level and increased mortality in hip fracture patients [22,30], we included this parameter in the current study. However, we failed to discover heamoglobin level as a predictor of one-year mortality in this patient group.

Mortality rate observed in this study is comparable with those reported in literature for hip fracture where the one-year mortality range from $8.4 \%$ to $36 \%$ [21]. One prospective study among elderly women with a hip fracture was conducted to determine whether the survival outcome differed by fracture type. They revealed one-year mortality in intertrochanteric fracture patients at $28 \%$ which was markedly higher than their $11 \%$ mortality in femoral neck fracture patients [24]. The relationship of fracture type and mortality in geriatric hip fracture patients was not specifically addressed in a the systematic review by Butler et al [31]. Thus, the impact of fracture type to postoperative mortality in hip fracture patients needs further evaluation. Several investigators have demonstrated the predictive value of serum albumin and TLC on postoperative mortality in hip fracture patients. Koval et al [16] found that an albumin level below $35.0 \mathrm{~g} / \mathrm{L}$ was predictive for increased length of stay and in-hospital mortality and a total lymphocyte count below $1.50 \times 10^{9}$ cells/L was predictive for oneyear mortality. They also reported that patients with abnormal albumin and total lymphocyte counts were 3.9 times more likely to die within one year after surgery. In a similar study by 0'Daly et al, survival analysis of 200 patients with hip fracture showed patients with low albumin and TLC levels were more likely to die within 12 months but only serum albumin and age were found to be significant independent prognostic factors [14]. On the contrary, a study from Singapore evaluating the risk factors for mortality in elderly patients with hip fractures revealed patients with low albumin and TLC level had a lower mean survival time. However, the albumin level lost its predictive value of mortality after multivariate analysis [28]. Recently, a systematic review and meta-analysis concluded that low albumin and TLC just had moderate or limited evidence in predicting mortality following hip fracture surgery [6] which indicates that further studies are needed to reevaluate their predictive values.

Based on the ROC curve results, we get an optimal cut-off level 
of $30.0 \mathrm{~g} / \mathrm{L}$ for albumin and $1.0 \times 10^{9}$ cells/L for TLC, respectively. It helps to identify, pre-operatively, those patients who will be at high risk following surgery. In a prospective study, Foster et al. [19] reported a mortality rate of $70 \%$ in hip fracture patients with albumin less than $30.0 \mathrm{~g} / \mathrm{L}$, compared with a mortality rate of $18 \%$ in those $\geq 30.0 \mathrm{~g} / \mathrm{L}$. They suggested a more aggressive approach to nutritional support for those with a serum albumin below $30.0 \mathrm{~g} / \mathrm{L}$. Similar results were obtained later in another prospective cohort study including 248 hip fracture patients aged 70 and older, in which Pioli et al found that albumin $30.0 \mathrm{~g} / \mathrm{L}$ was the only significant predictor of in-hospital mortality [20]. More recently, a survival study in Japan revealed a different albumin cut-off point of $36.0 \mathrm{~g} / \mathrm{L}$ for predicting the 4-year postoperative mortality in hip fracture patients [21]. However, the patients included in this study were younger and seemed having a good nutrition situation with a mean albumin level of $35.4 \mathrm{~g} / \mathrm{L}$. We believe that the different health level of the patients leads to the different cut-off point value and the warning line should be established based on the local data.

There are several limitations in this study. First, it is a retrospective study, and thus completeness of medical records was limited with regard to preoperative co-morbidities, functional status and cognitive level which were found to be significant factors of one year mortality [3,20,22]. Future studies should include a comprehensive assessment of patient characteristics to investigate the strength of albumin and TLC as predictors of mortality in hip fractured patients. The unmeasured differences in medical therapies may contribute to the results. Likewise, nutritional intervention is also very important. It has been shown to modify nutritional parameters and albumin levels, but it still not regularly managed for the hip fractured patients in our hospital. The impaction of surgical delay was not included, because surgical decision does not always completely depend on the patients' condition. In the health delivery system of China, patients sometimes had to wait for an extra bed before admission, even for an emergency traumatic case. Sometimes the surgery had to be postponed, because all members of the patient's family did not reach a consensus. The multivariate Cox regression should be a better statistical approach for survival analysis if we can get the exact death time of the patients. We did not measure functional outcome which is essential for a fracture repair. The relationship between the nutritional status and functional recovery will be studied in the future and is not the purpose of this study.

In conclusion, serum albumin and TLC are useful clinical markers for nutritional assessment and effective predictors of one-year mortality in aged patients with intertrochanteric fracture. In combination, they provide valuable information, helping to identify those patients who are candidates for interventions to reduce their risk of premature death. Whether a strategy of albumin treatment or nutritional supplementation could bring benefit for improving the survival rate in these highrisk patients warrants further evaluation.

\section{Acknowledgment}

The authors acknowledge the works of professor Ray
Vanderby Jr,( Department of Orthopedics and Rehabilitation, University of Wisconsin School of Medicine and Public Health) as an English reviewer of this paper. There is no grant or other funding to support this study. The authors confirm that there is no conflict of interest in the preparation of this article.

\section{References}

1. Roberts SE, Goldacre MJ (2003) Time trends and demography of mortality after fractured neck of femur in an English population, 1968-98: database study. BMJ 327(7418): 771-775.

2. Abrahamsen B, van Staa T, Ariely R, Olson M, Cooper C (2009) Excess mortality following hip fracture: a systematic epidemiological review. Osteoporos Int 20(10): 1633-1650.

3. Kannegaard PN, van der Mark S, Eiken P, Abrahamsen B (2010) Excess mortality in men compared with women following a hip fracture. National analysis of comedications, comorbidity and survival. Age Ageing 39(2): 203-209.

4. Johnell O, Kanis JA (2004) An estimate of the worldwide prevalence, mortality and disability associated with hip fracture. Osteoporos Int 15(11): 897-902.

5. Kanis JA, Oden A, Johnell O, De Laet C, Jonsson B, et al. (2003) The components of excess mortality after hip fracture. Bone 32(5): 468-473.

6. Hu F1, Jiang C, Shen J, Tang P, Wang Y (2012) Preoperative predictors for mortality following hip fracture surgery: a systematic review and metaanalysis. Injury 43(6): 676-685.

7. Lumbers M1, New SA, Gibson S, Murphy MC (2001) Nutritional status in elderly female hip fracture patients: comparison with an age-matched home living group attending day centres. Br J Nutr 85(6): 733-740.

8. Eneroth M, Olsson UB, Thorngren KG (2005) Insufficient fluid and energy intake in hospitalised patients with hip fracture. A prospective randomised study of 80 patients. Clin Nutr 24(2): 297-303.

9. Perez Durillo FT, Ruiz Lopez MD, Bouzas PR, Martin-Lagos A (2010) [Nutritional status in elderly patients with a hip fracture]. Nutr Hosp 25(4): 676-681.

10. Bonjour JP1, Schurch MA, Rizzoli R (1996) Nutritional aspects of hip fractures. Bone 18(3): 139S-144S.

11. Huang Z, Himes JH, McGovern PG (1996) Nutrition and subsequent hip fracture risk among a national cohort of white women. Am J Epidemiol 144(2): 124-134.

12. Murphy MC, Brooks CN, New SA, Lumbers ML (2000) The use of the Mini-Nutritional Assessment (MNA) tool in elderly orthopaedic patients. Eur J Clin Nutr 54(7): 555-562.

13. Guo JJ1, Yang H, Qian H, Huang L, Guo Z et al. (2010) The effects of different nutritional measurements on delayed wound healing after hip fracture in the elderly. J Surg Res 159(1): 503-508.

14. O’Daly BJ, Walsh JC, Quinlan JF, Falk GA, Stapleton R, et al. (2010) Serum albumin and total lymphocyte count as predictors of outcome in hip fractures. Clin Nutr 29(1): 89-93.

15. Van Hoang H, Silverstone FA, Leventer S, Wolf-Klein GP, Foley CJ (1998 ) The effect of nutritional status on length of stay in elderly hip fracture patients. J Nutr Health Aging 2(3): 159-161.

16. Koval KJ, Maurer SG, Su ET, Aharonoff GB, Zuckerman JD (1999) The effects of nutritional status on outcome after hip fracture. J Orthop Trauma 13(3): 164-169.

17. Lavernia CJ, Sierra RJ, Baerga L (1999) Nutritional parameters and short 
term outcome in arthroplasty. J Am Coll Nutr 18(3): 274-278.

18. Lee HP, Chang YY, Jean YH, Wang HC (2009) Importance of serum albumin level in the preoperative tests conducted in elderly patients with hip fracture. Injury 40(7): 756-759.

19. Foster MR, Heppenstall RB, Friedenberg ZB, Hozack WJ (1990) A prospective assessment of nutritional status and complications in patients with fractures of the hip. J Orthop Trauma4 (1): 49-57.

20. Pioli G, Barone A, Giusti A, Oliveri M, Pizzonia M, Razzano M, et al. (2006) Predictors of mortality after hip fracture: results from 1-year follow-up. Aging Clin Exp Res 18(5): 381-387.

21. Miyanishi K, Jingushi S, Torisu T (2010) Mortality after hip fracture in Japan: the role of nutritional status. J Orthop Surg (Hong Kong) 18(3): 265-270.

22. Ozturk A Ozkan Y, Akgöz S, Yalçýn N, Ozdemir RM et al. (2010)The risk factors for mortality in elderly patients with hip fractures: postoperative one-year results. Singapore Med J 51(2): 137-143.

23. Maffulli N, Dougall TW, Brown MT, Golden MH (1999) Nutritional differences in patients with proximal femoral fractures. Age Ageing 28(5): 458-462.

24. Haentjens P, Autier P, Barette M, Venken K, Vanderschueren D et al. (2007) Survival and functional outcome according to hip fracture type: a one-year prospective cohort study in elderly women with an intertrochanteric or femoral neck fracture. Bone 41(6): 958-964
25. Schipper IB, Steyerberg EW, Castelein RM, van der Heijden FH, den Hoed PT et al. (2004)Treatment of unstable trochanteric fractures. Randomised comparison of the gamma nail and the proximal femoral nail. J Bone Joint Surg Br 86(1): 86-94.

26. McNeil BJ, Keller E, Adelstein SJ (1975) Primer on certain elements of medical decision making. N Engl J Med 293(5): 211-215.

27. Gullberg B, Johnell O, Kanis JA (1997)World-wide projections for hip fracture. Osteoporos Int 7(5): 407-413.

28. Fox KM, Magaziner J, Hebel JR, Kenzora JE, Kashner TM et al. (1999) Intertrochanteric versus femoral neck hip fractures: differential characteristics, treatment, and sequelae. J Gerontol A Biol Sci Med Sci 54(12): 635-640.

29. Muraki S, Yamamoto S, Ishibashi H, Nakamura K (2006) Factors associated with mortality following hip fracture in Japan. J Bone Miner Metab 24(2): 100-4.

30. Gruson KI, Aharonoff GB, Egol KA, Zuckerman JD, Koval KJ (2002) The relationship between admission hemoglobin level and outcome after hip fracture. J Orthop Trauma, 16(1): 39-44.

31. Butler M, Forte ML, Joglekar SB, Swiontkowski MF, Kane RL (2011) Evidence summary: systematic review of surgical treatments for geriatric hip fractures. J Bone Joint Surg Am 93(12): 1104-1115. 